\title{
Contemporary technique of intraoperative 3-dimensional ultrasonography-guided transperineal prostate cryotherapy
}

\author{
Venu Chalasani, MBBS; ${ }^{*}$ Lori Gardi, BS; ${ }^{\dagger}$ Carlos H. Martinez, MD; ${ }^{*}$ Donal B. Downey, MD; ${ }^{\text {tt }}$ \\ Aaron Fenster, PhD; ${ }^{\text {}}$ Joseph L. Chin, MD*
}

See related article on page 142

\begin{abstract}
Successful cryotherapy of the prostate for neoplasms relies on imaging to achieve good oncological outcomes with minimal complications. Traditional prostatic cryotherapy relies on 2-dimensional ultrasonography (2DUS) guidance, which often makes it difficult to track the passage of needles in an oblique plane. We describe our initial 3-dimensional ultrasonography (3DUS) system, and the subsequent improvements that have been made during the last 10 years. Our imaging system uses a Philips HDI 5000 ultrasonography unit, a standard PC, a Matrox Meteor II video frame grabber and 3DUS developed at Robarts Research Institute. For the cryotherapy we use ultrathin (17-gauge) IceRod needles. After image acquisition, preplanning is performed using the 3-dimensional (3D) software, and then the IceRod needles are inserted into the prostate. As the freezing process commences, continuous 3DUS images are taken and analyzed during the double freeze-thaw cycles to monitor the progress of the ice ball formation. Real-time intraoperative 3D imaging of the prostate during cryotherapy has allowed us to accurately preplan and then monitor the progression of ice ball formation, which represents a significant advantage over conventional 2DUS.
\end{abstract}

Can Urol Assoc J 2009;3(2):136-41

\section{Résumé}

Pour être efficace, la cryothérapie prostatique utilisée pour le traitement de tumeurs nécessite une technique d'imagerie en vue d'obtenir de bons résultats tout en minimisant les complications. La cryothérapie prostatique classique est guidée par échographie transrectale en deux dimensions, avec laquelle il est souvent difficile de suivre le trajet des aiguilles sur un plan oblique. Nous décrivons ici notre système d'échographie en trois dimensions initial, et les améliorations ultérieures apportées au cours des 10 dernières années. Notre système d'imagerie comprend un appareil d'échographie Philips HDI 5000, un ordinateur standard, un saisisseur d'image Matrox Meteor II et un logiciel d'échographie en trois dimensions élaboré au Robarts Research Institute; pour la cryothérapie, nous utilisons des aiguilles de calibre 17 de Galil Medical. Une fois l'imagerie en fonction, la planification est effectuée à l'aide du logiciel en 3D, puis les aiguilles de cryothérapie sont insérées dans la prostate. Dès le début du processus de congélation, des images en 3D sont continuellement produites et analysées pendant les cycles de congélation et de décongélation afin de suivre la formation de la masse gelée. L'imagerie peropératoire en 3D et en temps réel de la prostate pendant la cryothérapie nous permet de planifier de façon précise et de suivre la formation de la masse gelée, et constitue un avantage important par rapport à la méthode classique d'échographie transrectale en deux dimensions.

\section{Introduction}

Prostate cryosurgery was first reported by Gonder and colleagues $^{1,2}$ in the 1960 s as a minimally invasive method of treating bladder outlet obstruction caused by benign prostatic hyperplasia and prostate cancer. However, the inability to monitor the position of the probe and control the ice ball formation led to a high rate of urethrorectal fistula and urethral tissue sloughing. ${ }^{3}$ The procedure did not gain popularity because of these serious technical limitations. Several technical advances in the early 1990s led to the resurgence of interest in the procedure, including improved endourological percutaneous techniques, the introduction of real-time transrectal ultrasonography, and improvement in cryogenic technology and safety monitoring devices such as thermal sensors and urethral warming devices., ${ }^{4,5}$

The new generation of cryosurgery machines exploits the Joule-Thomson effect, in which different gases undergo unique temperature changes when depressurized, according to unique gas coefficients. Further improvements in technology include a significant reduction in probe diameter. Ultrathin probes with sharp tips now permit direct transperineal probe placement through a conventional brachytherapy-type template without the need for tract dilation. ${ }^{6-8}$ Encouraging results using this third-generation cryotechnology have been reported. .,10 $^{2}$

Current prostate cryotherapy is a minimally invasive treatment for prostate cancer, which involves percutaneous insertion of 5-8 cryoprobes into the prostate for rapid freezing, 
using conventional 2-dimensional ultrasonography (2DUS) for guiding the cryoprobes into the prostate and for monitoring the prostate freeze-thaw cycle. Conventional 2DUS allows the real-time tracking of parallel cryoprobe insertion; however, it does not provide enough information for accurate planning of insertion paths or live monitoring of trajectories oblique to the 2-dimensional (2D) image plane. In the mid-1990s, we incorporated 3-dimensional ultrasonography (3DUS) into our intraoperative cryotherapy program, using software developed at Robarts, which gave us the ability to capture the geometry of the prostate gland and "plan" the cryoprobe insertion paths (in a primitive manner) as well as track nonparallel cryoprobe trajectories during insertion. After insertion of the cryoprobes, 3DUS assists with placement verification. ${ }^{11,12}$

This original system involved a 20-second delay with data acquisition and reconstruction, and provided position verification after the cryoprobe insertion. When malalignment or malpositioning of the cryoprobess was detected, they would have to be withdrawn and the "blind" insertion process repeated, followed by repeat scanning. The updated system allows detailed preplanning and accurate targeting before the actual needle insertion. The system permits real-time monitoring of the freezing process in all 3 planes and encroachment of the ice balls on crucial structures (i.e., rectal wall and external urinary sphincter).

The updated system complements the improvement in cryoprobe design as ultrathin 17-gauge $(1.47 \mathrm{~mm})$ IceRod (Galil Medical) needles are used in place of the larger cryoprobes (Cryocare system) used previously.

\section{Technique}

A Philips HDI 5000 (Philips Medical Systems) ultra-
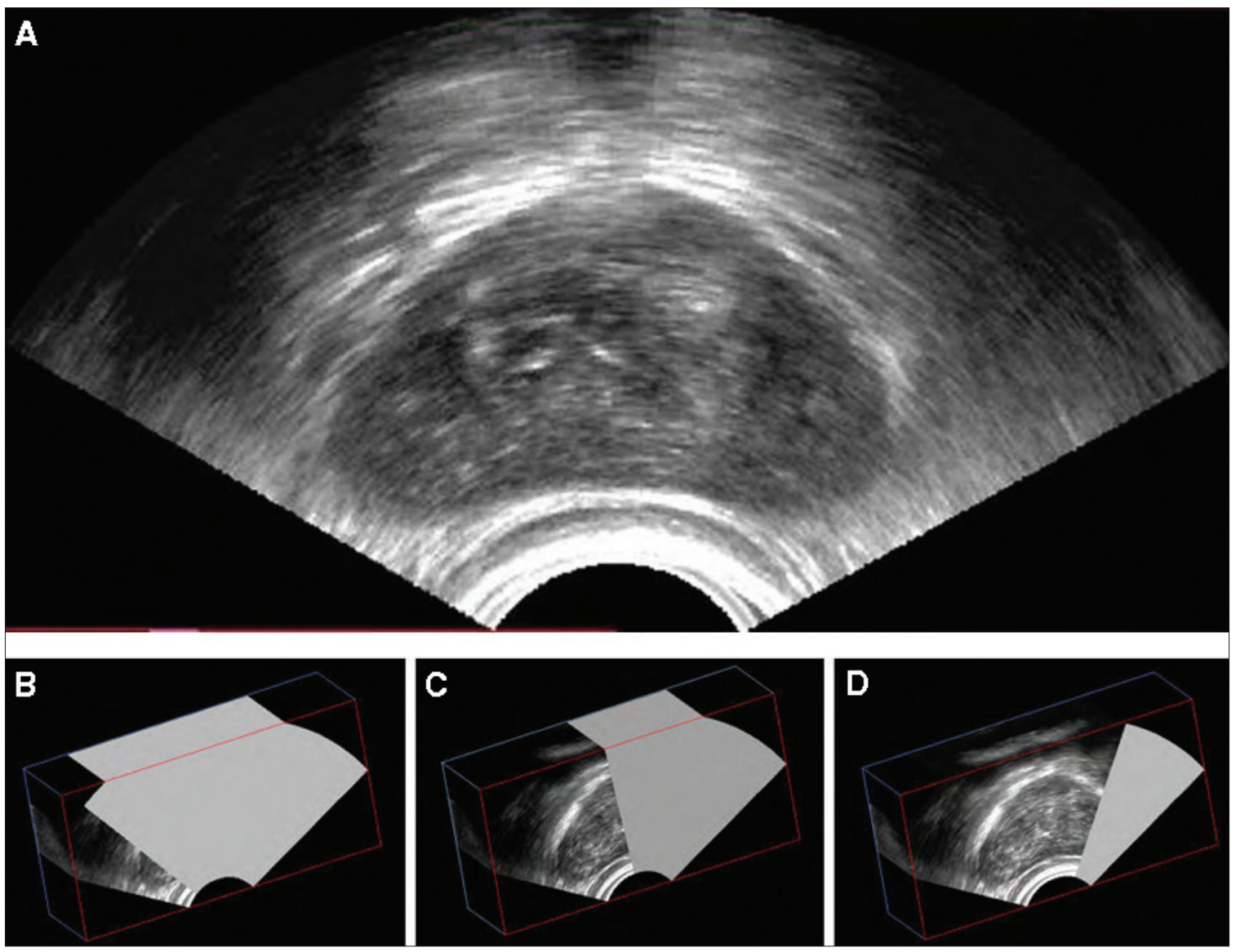

Fig. 1. Conventional coronal image of the prostate using 2-dimensional (2D) ultrasonography (A). Acquiring and storing $2 \mathrm{D}$ images of the prostate to create a 3-dimensional image $(B, C, D)$. 
sonography unit with a transrectal probe, and a standard PC computer are wheeled into the operating room and connected together via a Matrox Meteor II video frame grabber (Matrox Electronic Systems, Ltd.). We use 3DUS computer software developed at Robarts Research Institute. ${ }^{11}$ A Philips biplanar transrectal probe (Philips Medical Systems) is attached to a custom computer-controlled rotational mover device, which is then fastened to the operating room bed using an RTP 6000 stabilizer unit (Radiation Therapy Products). Under general or spinal anesthesia, the patient is placed in the lithotomy position. A cystotomy is performed using a 10-Fr. tocar percutaneous insertion set. The transrectal ultrasonography probe is then maneuvered into the rectum until the prostate can be clearly seen in the transverse 2DUS image. The linear array of the biplanar transrectal probe is used to acquire a series of sagittal $2 \mathrm{D}$ images while the probe is mechanically rotated through about 100 degrees around the long axis of the probe via computer control (Fig. 1). The images are stored in the corresponding position in the 3DUS image and visualized as a 3DUS image in real time. Once the 3DUS image of the prostate is complete, the placement of the IceRod needles can be planned. A template is displayed in the transverse view of the 3DUS image indicating where the IceRod needles can be targeted by the surgeon (Fig. 2). The surgeon maps out the respective probe positions, leaving appropriate distances between probes, to the lateral prostate boundaries, to the urethra and to Denonvilliers fascia. For each IceRod position selected, the rotational mover automatically and immediately moves to the correct sagittal plane such that the surgeon can follow his or her needle insertion in the 2DUS image (Fig. 3). It is also possible to use 3DUS tracking for oblique trajectories, which would be a challenge to visualize using 2DUS. Satisfactory probe positioning in all planes is promptly verified with $3 \mathrm{D}$ reconstructed images. Following placement of all IceRod needles, a final 3DUS image is taken and the actual insertion paths are further analyzed (Fig. 4). Particular attention is paid to the coronal plane and spatial relationship of the IceRod needles relative to one another, to the urethra and to the Denonvilliers fascia. Three thermocouples are then inserted under 3DUS guidance, at the apex, prerectal and intraprostatic locations. Flexible cystoscopy is repeated at this point to ensure integrity of the urethra. A urethral warming device is then inserted.

As the freezing process commences, continuous 3DUS images are taken and analyzed during the double freeze-thaw cycles to monitor the progress of the ice ball formation (Fig. 5). These real-time images permit vigilant monitoring of the freezing process in all 3 planes, with special attention paid to the progression of ice ball coverage of the entire prostate and, more importantly, proximity to the rectal wall and external sphincter.

\section{Discussion}

Continuing developments of the prostate cryoablation and imaging techniques have led to further improvement in efficacy and safety for clinical

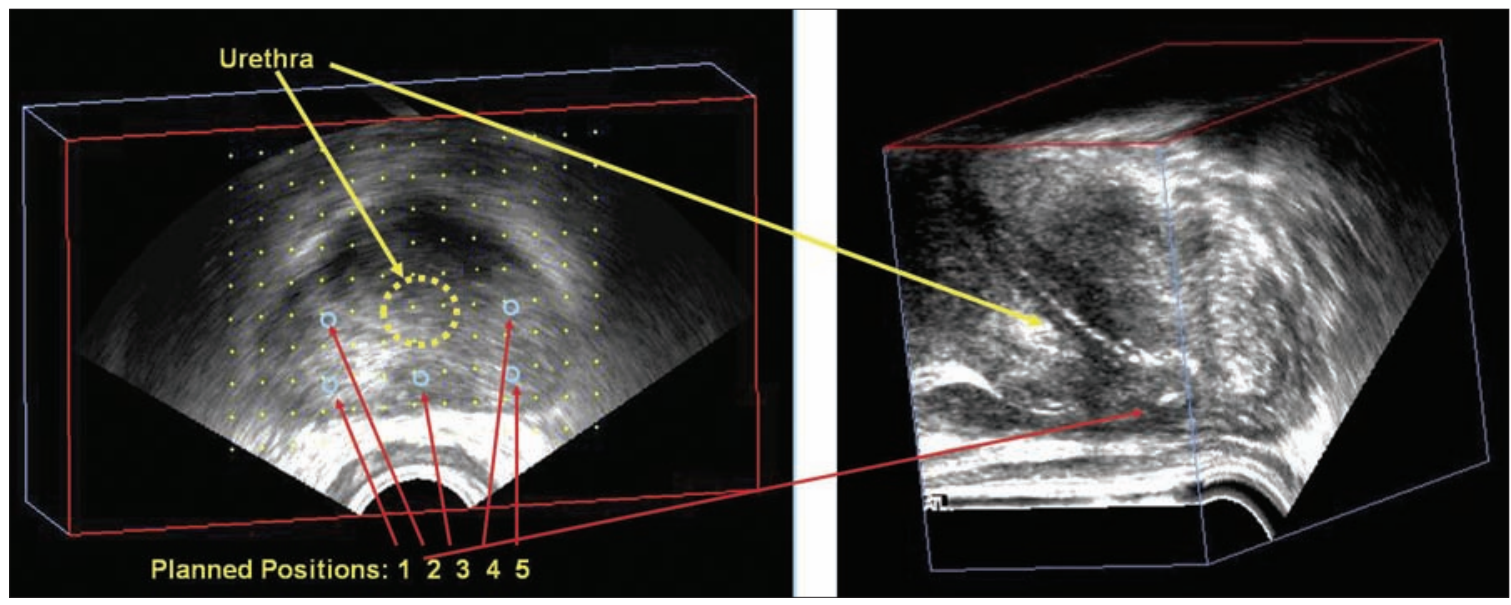

Fig. 2. Planning insertion of cryoprobes with 3-dimensional (3D) ultrasonography. Coronal (A) and sagital (B) 3D prostate images. 
use. Proper positioning and alignment of the cryoprobes is essential for complete prostate ablation and for preventing serious complications such as fistula formation and urinary incontinence. ${ }^{13,14}$ One of the difficulties during cryoprobe insertion is visualizing the tip of the needle if it passes into an oblique trajectory in real time. Wei and colleagues ${ }^{15}$ have developed an algorithm to segment and track brachytherapy needles inserted along oblique trajectories. This algorithm is applicable for all percutaneous interventions, including prostate cryotherapy, prostate biopsy and prostatic brachytherapy. In their approach, the reconstructed oblique (sagittal and coronal) planes containing the needle can be used to monitor the needle insertion in near real time, ${ }^{15}$ and promising results have been reported. ${ }^{16}$

A new slice-based 3D prostate segmentation method based on a continuity constraint, implemented as an autoregressive model, has recently been described. ${ }^{17}$ This new technique is an

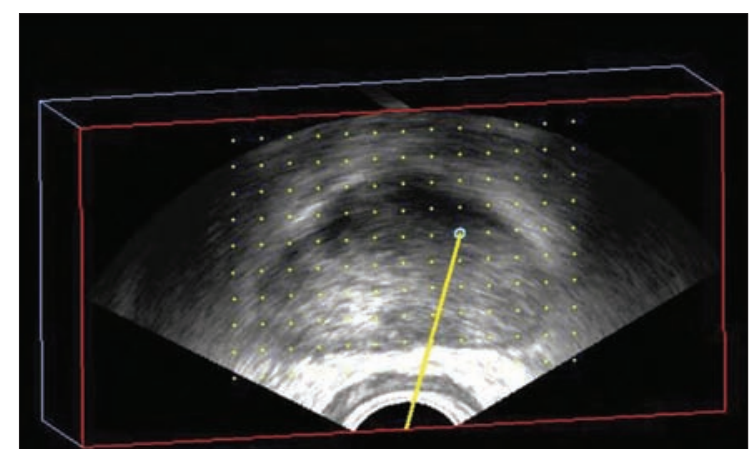

3DUS Transverse View Showing Mover Position

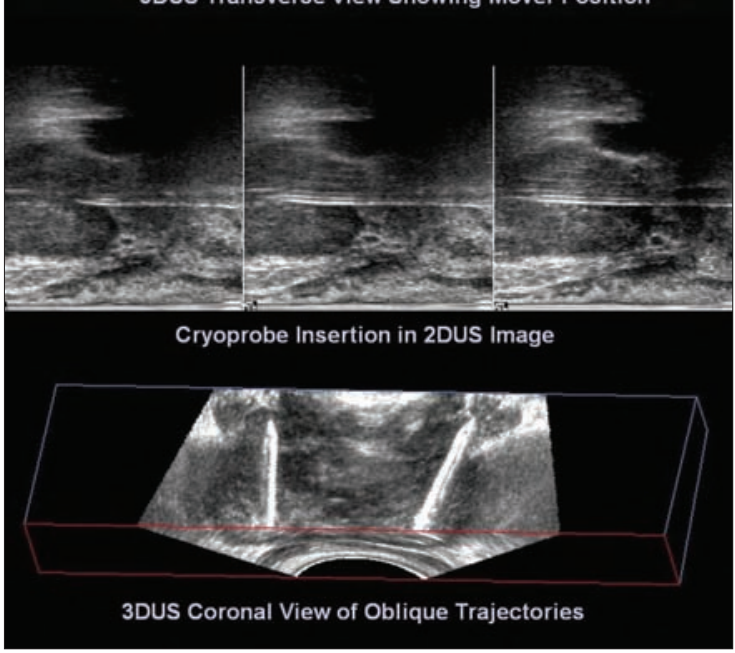

Fig. 3. Insertion of cryoprobes with 2-dimensional and 3-dimensional ultrasonography (2DUS, 3DUS) tracking. improvement from the standard slice-based 3D prostate segmentation method originally proposed by Wang and colleagues, ${ }^{18}$ which suffered from the effect of accumulation errors.

Our current system permits careful preplanning and targeting of probe placement with verification in 3 dimensions, as well as real-time monitoring of the freezing process. The intraoperative process is streamlined and time efficient and provides crucial safety checks for the cryoablation procedure. In our most recent 25 cases, we have found that the updated 3DUS greatly facilitated placement compared with the earlier set-up. In due course, once long-term follow-up data become available, we will be able to compare our long-term results using the 3DUS system with that of other centres that use 2DUS. A recent study at our centre comparing computed tomography, $T_{2}$-weighted magnetic resonance imaging (MRI) and 3DUS in calculating prostate volume found that 3DUS and MRI displayed the smallest variability and the

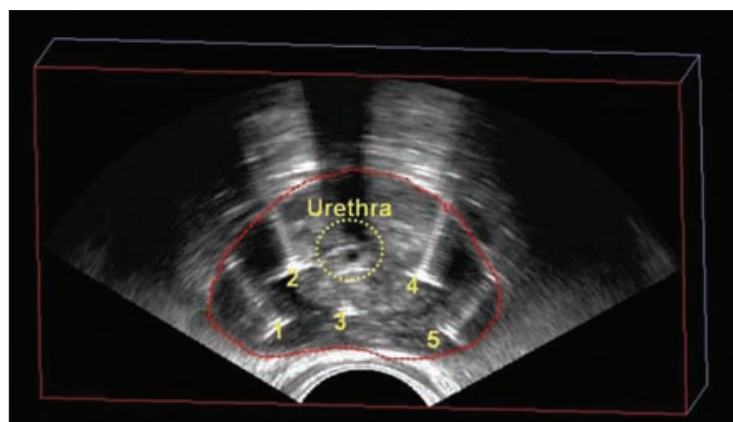

3DUS Transverse View of 5 Cryoprobes and Urethra

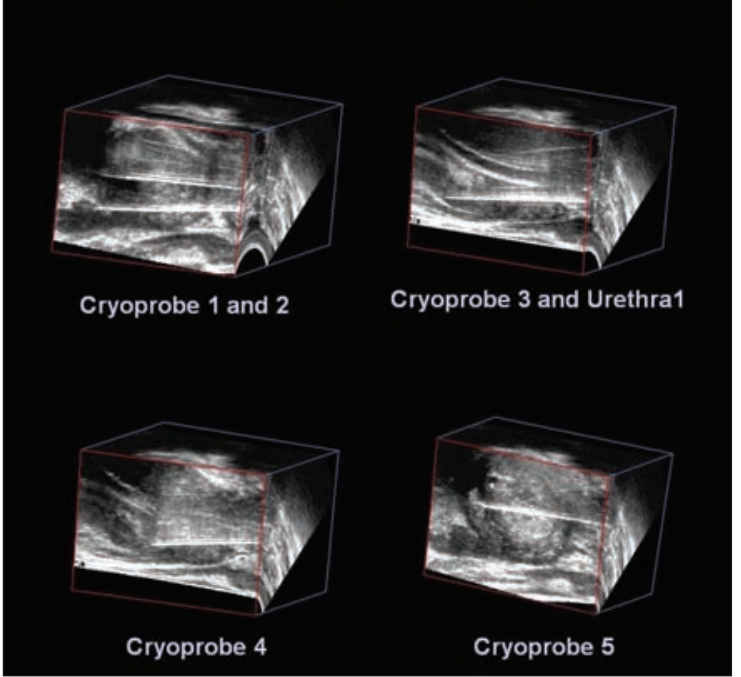

Fig. 4. Verifying with 3-dimensional ultrasonography (3DUS) cryoprobes positions. 


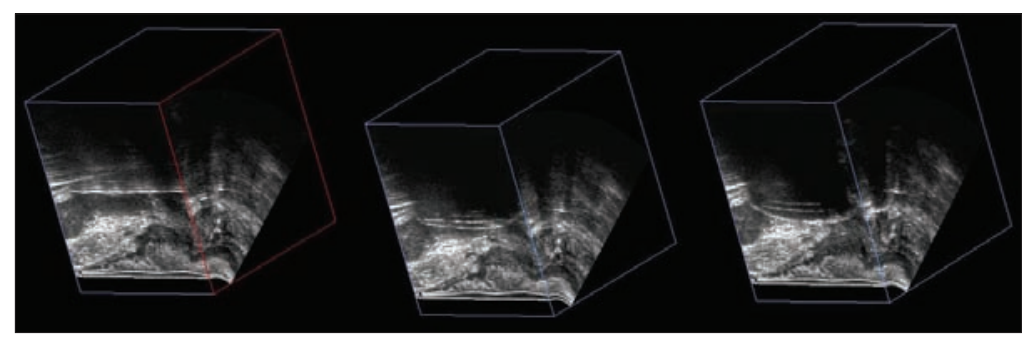

Fig. 5. Monitoring of the ice ball formation.

closest correspondence. ${ }^{19}$ From a practical viewpoint, using MRI to guide cryosurgery would be cumbersome compared with the simplicity and widespread availability of ultrasonography.

The future holds the potential for focal prostatic therapy as functional imaging continues to improve. Functional imaging such as the aforementioned 3DUS will be used to accurately localize the bulk of the prostatic cancer through biopsy $^{20}$ and assessment of radiological stage. ${ }^{21-23}$ It will then be possible to use 3DUS to guide focal therapies such as prostate cryotherapy, ${ }^{24-26}$ which will be aided by automated computerized planning ${ }^{27,28}$ and techniques such as $3 \mathrm{D}$ bubble packing. ${ }^{29}$ Furthermore, cryotechnology is likely to continue improving, with smaller needles leading to decreased morbidity. ${ }^{30}$

\section{Conclusion}

The use of 3DUS combined with real-time 2DUS offers an important advantage over conventional 2DUS cryotherapy procedures. With 3DUS, we have the ability to preplan the cryoprobe insertion paths, track the insertion process, verify the locations in 3 dimensions relative to the urethra and other important anatomy and to monitor the progress of the ice ball formation in any $3 \mathrm{D}$ plane in real time. This technology deserves further evaluation for integration into current image-guided therapy modalities for prostate cancer.

From the *Division of Urology, University of Western Ontario, London, Ont., †Imaging Research Laboratories, Robarts Research Institute, London, Ont., and the $\ddagger$ Department of Diagnostic Radiology and Nuclear Medicine, University of Western Ontario, London, Ont.

Acknowledgements: The authors acknowledge the financial support from the Ontario Research Development Fund and the Canadian Institutes of Health Research.

This article has been peer reviewed.

Competing interests: None declared.

\section{References}

1. Gonder MJ, Soanes WA, Smith V. Chemical and morphologic changes in the prostate following extreme cooling. Ann NY Acad Sci. 1965;125:716-29.

2. Gonder MJ, Soanes WA, Smith V. Experimental prostate cryosurgery. Invest Urol 1964; 1:610-9.

3. Flocks RH, Nelson CM, Boatman DL. Perineal cryosurgery for prostatic carcinoma. J Urol 1972;108:933-5.

4. Onik G, Porterfield B, Rubinsky B, et al. Percutaneous transperineal prostate cryosurgery using transrectal ultrasound guidance: animal model. Urology 1991;37:277-81.

5. Onik GM, Cohen JK, Reyes GD, et al. Transrectal ultrasound-guided percutaneous radical cryosurgical ablation of the prostate. Cancer 1993;72:1291-9.

6. Han KR, Cohen JK, Miller RJ, et al. Treatment of organ confined prostate cancer with third generation cryosurgery: preliminary multicenter experience. J Urol 2003;170:1126-30.

7. Zisman A, Leibovici D, Siegel Yl, et al. Prostate cryoablation without an insertion kit using direct transperineal placement of ultrathin freezing probes. Tech Urol 2000;6:34-6.

8. Zisman A, Pantuck AJ, Cohen JK, et al. Prostate cryoablation using direct transperineal placement of ultrathin probes through a 17-gauge brachytherapy template-technique and preliminary results. Urology 2001;58:988-93.

9. Hubosky SG, Fabrizio MD, Schellhammer PF, et al. Single center experience with thirdgeneration cryosurgery for management of organ-confined prostate cancer: critical evaluation of short-term outcomes, complications, and patient quality of life. J Endourol 2007;21:1521-31.

10. Polascik TJ, Nosnik I, Mayes JM, et al. Short-term cancer control after primary cryosurgical ablation for clinically localized prostate cancer using third-generation cryotechnology. Urology 2007;70:117-21.

11. Chin JL, Downey DB, Onik G, et al. Three-dimensional prostate ultrasound and its application to cryosurgery. Tech Urol 1996;2:187-93.

12. Chin JL, Downey DB, Mulligan M, et al. Three-dimensional transrectal ultrasound guided cryoablation for localized prostate cancer in nonsurgical candidates: a feasibility study and report of early results. J Urol 1998;159:910-4.

13. Chin JL, Pautler SE, Mouraviev V, et al. Results of salvage cryoablation of the prostate after radiation: identifying predictors of treatment failure and complications. J Urol 2001;165:1937-41

14. Ng CK, Moussa M, Downey DB, et al. Salvage cryoablation of the prostate: followup and analysis of predictive factors for outcome. J Urol 2007;178:1253-7.

15. Wei Z, Gardi L, Downey DB, et al. Oblique needle segmentation and tracking for 3D TRUS guided prostate brachytherapy. Med Phys 2005;32:2928-41.

16. Ding $M$, Cardinal HN, Fenster A. Automatic needle segmentation in three-dimensional ultrasound images using two orthogonal two-dimensional image projections. Med Phys 2003;30:222-34.

17. Ding M, Chiu B, Gyacskov I, et al. Fast prostate segmentation in $3 D$ TRUS images based on continuity constraint using an autoregressive model. Med Phys 2007;34:4109-25.

18. Wang Y, Cardinal HN, Downey DB, et al. Semiautomatic three-dimensional segmentation of the prostate using two-dimensional ultrasound images. Med Phys 2003;30:887-97.

19. Smith WL, Lewis C, Bauman $G$, et al. Prostate volume contouring: a $3 D$ analysis of segmentation using 3DTRUS, CT, and MR. Int J Radiat Oncol Biol Phys 2007;67:1238-47.

20. Barzell WE, Melamed MR. Appropriate patient selection in the focal treatment of prostate cancer: the role of transperineal 3-dimensional pathologic mapping of the prostate 
- a 4-year experience. Urology 2007;70:27-35.

21. Baumann M, Mozer P, Daanen V, et al. Towards 3D ultrasound image based soft tissue tracking: a transrectal ultrasound prostate image alignment system. Med Image Comput Comput Assist Interv Int Conf Med Image Comput Comput Assist Interv 2007:10:26-33.

22. Long JA, Daanen V, Moreau-Gaudry A, et al. Prostate biopsies guided by three-dimensional real-time (4-D) transrectal ultrasonography on a phantom: comparative study versus two-dimensional transrectal ultrasound-guided biopsies. Eur Urol 2007;52:1097-104.

23. Mitterberger $M$, Pinggera $G M$, Pallwein $L$, et al. The value of three-dimensional transrectal ultrasonography in staging prostate cancer. BJU Int 2007;100:47-50.

24. Ellis DS, Manny TB Jr, Rewcastle JC. Focal cryosurgery followed by penile rehabilitation as primary treatment for localized prostate cancer: initial results. Urology 2007;70:9-15.

25. Lambert EH, Bolte K, Masson P, et al. Focal cryosurgery: encouraging health outcomes for unifocal prostate cancer. Urology 2007;69:1117-20.

26. Onik G, Vaughan D, Lotenfoe R, et al. "Male lumpectomy": focal therapy for prostate cancer using cryoablation. Urology 2007;70:16-21.

27. Tanaka D, Shimada K, Rossi MR, et al. Computerized planning of prostate cryosurgery with pullback operation. Comput Aided Surg 2008;13:1-13.

28. Tanaka D, Shimada K, Rossi MR, et al. Towards intra-operative computerized planning of prostate cryosurgery. Int I Med Robot 2007:3:10-9.

29. Tanaka D, Shimada K, Rossi MR, et al. Cryosurgery planning using bubble packing in 3D. Comput Methods Biomech Biomed Engin 2008;11:113-21.

30. Gowardhan B, Greene D. Cryotherapy for the prostate: an in vitro and clinical study of two new developments; advanced cryobneedles and a temperature monitoring system. BJU Int 2007;100:295-302.

Correspondence: Dr. Joseph Chin, Division of Urology, University of Western Ontario, London 0N N6A 5K8; Joseph.Chin@/hsc.on.ca

CUAJ is read by at least 3000 urologists and related specialists in Canada and around the world.

If you want to reach urologists, there is no better place for your classified advertisement. Please contact the journal at journal@cua.org or call 514 395-0376 x40 for a price list, specifications and deadlines. 